\title{
IMPACTS OF NOISE ON ORDINARY DIFFERENTIAL EQUATIONS
}

\author{
GUANGYING LV ${ }^{1}$, JINQIAO DUAN ${ }^{2}$, LIANG WANG ${ }^{3}$, AND JIANG-LUN WU \\ ${ }^{1}$ School of Mathematical Science \\ Nanjing Normal University \\ Nanjing, 210023, P.R. CHINA \\ ${ }^{2}$ Department of Applied Mathematics \\ Illinois Institute of Technology \\ Chicago, IL 60616, USA \\ ${ }^{3}$ Department of Applied Mathematics \\ Northwestern Polytechnical University \\ Xi'an, 710072, P.R. CHINA \\ ${ }^{4}$ Department of Mathematics \\ Swansea University \\ Swansea SA2 8PP, UK
}

\begin{abstract}
In this paper, we consider the impacts of noise on ordinary differential equations. We first prove that the weak noise can change the value of equilibrium and the strong noise can destroy the stability of equilibrium. Then we consider the competition between the nonlinear term and noise term, which shows that noise can induce singularities (finite time blow up of solutions) and that the nonlinear term can prevent the singularities. Besides that, some simulations are given in order to illustrate our results.
\end{abstract}

AMS Subject Classification: $35 \mathrm{~K} 20,60 \mathrm{H} 15,60 \mathrm{H} 40$

Received: February 3, 2017; Accepted: October 2, 2017;

Published: March 11, $2018 \quad$ doi: 10.12732 dsa.v27i2.2

Dynamic Publishers, Inc., Acad. Publishers, Ltd. https://acadsol.eu/dsa

\section{INTRODUCTION}

The theory of stochastic differential equations (SDEs) has been very well developed 
since the seminal work of the great mathematician Kiyosi Itô in the mid 1940s. Existence and uniqueness of solutions of SDEs have been extensively studied by many authors $[7,15]$ under the conditions that both dirt and diffusion coefficients satisfy linear growth and global Lipschitz condition. SDEs (as well as stochastic functional differential equations) with non-Lipschitzian coefficients have received much attention widely, see, e.g., [5, 6, 9, 10, 14], just mention a few. In the present paper, we aim to study the impact of noise on the solutions of ODEs, see [12].

Given a probability space $(\Omega, \mathcal{F}, P)$ endowed with a complete filtration $\left(\mathcal{F}_{t}\right)_{t \geq 0}$. For simplicity, we only consider the case that the image belongs to $\mathbb{R}$. That is, we consider the following problem

$$
d X_{t}=b\left(X_{t}\right) d t+\sigma\left(X_{t}\right) d W_{t}, \quad X_{0}=x \in \mathbb{R}
$$

where $W_{t}$ is white noise. In this paper, we focus on the effect of noise.

Firstly, in Section 2, we consider the following special case

$$
d X_{t}=\alpha\left(\beta-X_{t}^{2}\right) X_{t} d t+k(t) X_{t} d W_{t}, \quad X_{0}=x \in \mathbb{R}
$$

where $\alpha>0, \beta>0$ and $k(t)$ is a continuous function. In this case, we can write the explicit solution of (1.2) and thus we can prove the effect of noise clearly. We prove that weak noise, $\alpha \beta>\frac{k_{m}^{2}}{2}\left(k_{m}=\lim _{t \rightarrow \infty} \sqrt{\frac{1}{t} \int_{0}^{t} k^{2}(s) d s}\right)$, can change the value of equilibrium and strong noise, $\alpha \beta \leq \frac{k_{m}^{2}}{2}$, can destroy the stable of equilibrium, see [2] for similar results.

Secondly, in Section 3, the competition between nonlinear term and noise term will be investigated. Consider the following problem

$$
\left\{\begin{array}{l}
d X_{t}=\left(-k_{1} X_{t}^{\gamma}\right) d t+k_{2} X_{t}^{m} d W_{t}, \quad t>0 \\
X_{0}=x
\end{array}\right.
$$

where $k_{1} \geq 0, k_{2} \in \mathbb{R}, m \geq 1$ and $\gamma>1$ satisfying $(-1)^{\gamma}=-1$. It turns out that the noise can induce singularity (finite time blowup) and the nonlinearity can prevent the solution blowing up, see the reference $[3,4]$.

Lastly, apart from the analysis proof, we shall give some simulations in Section 4, which show that our results are right.

\section{A SPECIAL CASE}

In this section, we are interested in the effect of noise on equilibrium. The effect of noise on blowup time is also investigated. 
Now, we consider the following equation

$$
\left\{\begin{array}{l}
d X_{t}=\alpha\left(\beta-X_{t}^{2}\right) X_{t} d t+k(t) X_{t} d W_{t}, \quad t>0, \\
X_{0}=x
\end{array}\right.
$$

where $\alpha>0, \beta>0$ and $k(t)$ is a continuous function. Let $Y(t)=e^{-\int_{0}^{t} k(s) d W(s)} X(t)$. Itô formula implies that

$$
d Y(t)=\alpha\left(\beta-\frac{1}{2 \alpha} k^{2}(t)-e^{2 \int_{0}^{t} k(s) d W(s)} Y^{2}(t)\right) Y(t) d t .
$$

Set $Z(t)=e^{-\alpha \beta t+\frac{1}{2} \int_{0}^{t} k^{2}(s) d s} Y(t)$. The above equality gives

$$
Z^{2}(t)=\frac{1}{x^{-2}+2 \alpha \int_{0}^{t} e^{2\left[\left(\alpha \beta-\frac{1}{2 t} \int_{0}^{t} k^{2}(s) d s\right) t+\int_{0}^{t} k(s) d W(s)\right]} d t}
$$

Thus we have

$$
X^{2}(t)=\frac{e^{2\left[\left(\alpha \beta-\frac{1}{2 t} \int_{0}^{t} k^{2}(s) d s\right) t+\int_{0}^{t} k(s) d W(s)\right]}}{x^{-2}+2 \alpha \int_{0}^{t} e^{2\left[\left(\alpha \beta-\frac{1}{2 s} \int_{0}^{s} k^{2}(r) d r\right) s+\int_{0}^{s} k(r) d W(r)\right]} d s} .
$$

In particular, $k(t) \equiv 0,(2.2)$ becomes

$$
X^{2}(t)=\frac{e^{2 \alpha \beta t}}{x^{-2}+\beta^{-1}\left(e^{2 \alpha \beta t}-1\right)},
$$

which yields that

$$
\lim _{t \rightarrow \infty} X^{2}(t)=\beta
$$

Theorem 2.1. Let $X(t)$ be the solution of equation (2.1). If $\alpha \beta>\frac{k_{m}^{2}}{2}$, then for any $\varepsilon>0$, there exists $t_{0}>0$ such that

$P\left\{\left|\frac{1}{t} \int_{0}^{t} X^{2}(s) d s-\left(\beta-\frac{k_{m}^{2}}{2 \alpha}\right)\right|>\varepsilon\right.$ for some $\left.t>T\right\}<\exp \left\{-\frac{\alpha^{2} \varepsilon^{2} T^{2}}{32 \int_{0}^{T} k^{2}(r) d r}\right\}$

for all $T>t_{0}$, where $k_{m}=\lim _{t \rightarrow \infty} \sqrt{\frac{1}{t} \int_{0}^{t} k^{2}(s) d s}$. In particular, as $t \rightarrow \infty$,

$$
\frac{1}{t} \int_{0}^{t} X^{2}(s) d s \rightarrow \beta-\frac{k_{m}^{2}}{2 \alpha}
$$

almost surely. If $\alpha \beta \leq \frac{k_{m}^{2}}{2}$, then the solution $X(t) \rightarrow 0$ almost surely as $t \rightarrow \infty$.

Proof. The proof of this lemma is similar to [13, Lemma 2.1]. We only give the outline of the proof. From (2.2), it is easy to see that

$$
\frac{1}{t} \int_{0}^{t} X^{2}(s) d s=\frac{1}{2 \alpha} \log z(t)-\frac{1}{2 \alpha} \log z(0),
$$


where $z(t)=x^{-2}+2 \alpha \int_{0}^{t} e^{2\left[\alpha\left(\beta-\frac{k^{2}}{2 \alpha}\right) s+\int_{0}^{s} k(r) d W(r)\right]} d s$. Define

$$
\hat{z}(t)=2 \int_{0}^{t} e^{2\left[\alpha\left(\beta-\frac{1}{2 \alpha t} \int_{0}^{t} k^{2}(s) d s\right) s\right]} d s .
$$

Then it is easy to show that for any sufficiently small $\varepsilon>0$, there exists $t_{0}^{*}>0$ such that for $t>t_{0}^{*}$,

$$
k_{m}^{2}-\frac{1}{8} \alpha \varepsilon \leq \frac{1}{t} \int_{0}^{t} k^{2}(s) d s \leq k_{m}^{2}+\frac{1}{8} \alpha \varepsilon
$$

and

$$
\int_{0}^{t_{0}^{*}} e^{\left.2 \alpha \beta s-\int_{0}^{s} k^{2}(r) d r\right)} d s \leq M \exp \left(2 \alpha \beta t-k_{m}^{2} t+\frac{1}{8} \alpha \varepsilon t\right),
$$

where $M$ is a constant satisfying

$$
\log \left(\frac{1}{2 \alpha \beta-k_{m}^{2}+\frac{1}{8}}+M\right) \leq \frac{1}{8} \alpha \varepsilon t .
$$

On the other hand, there exists $\hat{t}>t_{0}^{*}$ such that for $t>\hat{t}$,

$$
\exp \left(2 \alpha \beta t_{0}^{*}-k_{m}^{2} t_{0}^{*}-\frac{1}{8} \alpha \varepsilon t_{0}^{*}\right) \leq \frac{1}{2} \exp \left(2 \alpha \beta t-k_{m}^{2} t-\frac{1}{8} \alpha \varepsilon t\right),
$$

and

$$
\log \left(\frac{1}{2\left(\alpha \beta-\frac{1}{2} k_{m}^{2}+\frac{1}{16} \alpha \varepsilon\right)}\right) \geq-\frac{1}{8} \alpha \varepsilon t
$$

Therefore, for $t>t_{0}^{*}$, it follows from (2.5) and (2.6) that

$$
\begin{aligned}
\hat{z}(t)= & 2 \int_{0}^{t} e^{2\left[\alpha\left(\beta-\frac{1}{2 \alpha t} \int_{0}^{t} k^{2}(s) d s\right) s\right]} d s \\
= & 2 \int_{0}^{t_{0}^{*}} e^{2\left[\alpha\left(\beta-\frac{1}{2 \alpha t} \int_{0}^{t} k^{2}(s) d s\right) s\right]} d s+2 \int_{t_{0}^{*}}^{t} e^{2\left[\alpha\left(\beta-\frac{1}{2 \alpha t} \int_{0}^{t} k^{2}(s) d s\right) s\right]} d s \\
\leq & 2 \int_{0}^{t_{0}^{*}} e^{2\left[\alpha\left(\beta-\frac{1}{2 \alpha t} \int_{0}^{t} k^{2}(s) d s\right) s\right]} d s+2 \int_{t_{0}^{*}}^{t} e^{2\left[\alpha\left(\beta-\frac{k_{m}^{2}}{2 \alpha}\right) s+\frac{1}{8} \alpha \varepsilon s\right]} d s \\
= & 2 \int_{0}^{t_{0}^{*}} e^{2\left[\alpha\left(\beta-\frac{1}{2 \alpha t} \int_{0}^{t} k^{2}(s) d s\right) s\right]} d s+\frac{1}{\alpha \beta-\frac{k_{m}^{2}}{2}+\frac{1}{8} \alpha \varepsilon} \\
& \times\left[\exp \left(2 \alpha \beta t-k_{m}^{2} t+\frac{1}{8} \alpha \varepsilon t\right)-\exp \left(2 \alpha \beta t_{0}^{*}-k_{m}^{2} t_{0}^{*}+\frac{1}{8} \alpha \varepsilon t_{0}^{*}\right)\right] \\
\leq & \left(\frac{1}{\alpha \beta-\frac{k_{m}^{2}}{2}+\frac{1}{8} \alpha \varepsilon}+M\right) \exp \left(2 \alpha \beta t-k_{m}^{2} t+\frac{1}{8} \alpha \varepsilon t\right) .
\end{aligned}
$$

Similarly, by (2.5) and (2.8), we have

$$
\hat{z}(t)=2 \int_{0}^{t} e^{2\left[\alpha\left(\beta-\frac{1}{2 \alpha t} \int_{0}^{t} k^{2}(s) d s\right) s\right]} d s
$$




$$
\begin{aligned}
\geq & 2 \int_{t_{0}^{*}}^{t} e^{2\left[\alpha\left(\beta-\frac{1}{2 \alpha t} \int_{0}^{t} k^{2}(s) d s\right) s\right]} d s \\
\geq & 2 \int_{0}^{t_{0}^{*}} e^{2\left[\alpha\left(\beta-\frac{k_{m}^{2}}{2 \alpha}\right) s-\frac{1}{8} \alpha \varepsilon s\right]} d s \\
= & \frac{1}{\alpha \beta-\frac{k_{m}^{2}}{2}+\frac{1}{8} \alpha \varepsilon} \\
& \times\left[\exp \left(2 \alpha \beta t-k_{m}^{2} t+\frac{1}{8} \alpha \varepsilon t\right)-\exp \left(2 \alpha \beta t_{0}^{*}-k_{m}^{2} t_{0}^{*}+\frac{1}{8} \alpha \varepsilon t_{0}^{*}\right)\right] \\
\geq & \frac{1}{2\left(\alpha \beta-\frac{k_{m}^{2}}{2}+\frac{1}{8} \alpha \varepsilon\right)} \exp \left(2 \alpha \beta t-k_{m}^{2} t+\frac{1}{8} \alpha \varepsilon t\right)
\end{aligned}
$$

Then taking logarithm to (2.10) and (2.11), it is easy to see from (2.7) and (2.9) that

$$
\left(2 \alpha \beta-k_{m}^{2}-\frac{1}{4} \alpha \varepsilon\right) t \leq \log \hat{z}(t) \leq\left(2 \alpha \beta-k_{m}^{2}+\frac{1}{4} \alpha \varepsilon\right) t .
$$

Recall that

$$
\frac{1}{\sqrt{\int_{0}^{t} k^{2}(r) d r}} \int_{0}^{s} k(r) d W_{r}, \quad 0 \leq s \leq t
$$

is a time changed Brownian motion $\chi\left(\frac{\int_{0}^{s} k^{2}(r) d r}{\int_{0}^{t} k^{2}(r) d r}\right)$. Here $\chi(u)$ is a standard Brownian motion with of time $u$. Therefore,

$$
Y(s)=\int_{0}^{s} k(r) d W_{r}=\sqrt{\int_{0}^{t} k^{2}(r) d r \chi}\left(\frac{\int_{0}^{s} k^{2}(r) d r}{\int_{0}^{t} k^{2}(r) d r}\right) .
$$

Let $C_{1}=\log (2 \alpha)$ and $C_{2}=\log \left(x^{-2}+2 \alpha\right)$. For any $\varepsilon>0$, take $t_{0} \geq \hat{t}$ such that

$$
\frac{\left|C_{1}-\log \left(x^{-2}\right)\right|}{\alpha t_{0}}<\varepsilon, \quad \frac{\left|C_{2}-\log \left(x^{-2}\right)\right|}{\alpha t_{0}}<\varepsilon .
$$

For any $T \geq t_{0}$, define

$$
\Omega_{T}=\left\{\omega \in \Omega:-\frac{\alpha \varepsilon T}{4 \sqrt{\int_{0}^{T} k^{2}(r) d r}}<\chi(u)<\frac{\alpha \varepsilon T}{4 \sqrt{\int_{0}^{T} k^{2}(r) d r}}, \text { for all } 0 \leq u \leq 1\right\} .
$$

Then from the well-known Doob's inequality (see $[11,13]$ )

$$
P\left(\Omega_{T}\right)>1-\exp \left(-\frac{\alpha^{2} \varepsilon^{2}}{32 \int_{0}^{T} k^{2}(r) d r} T^{2}\right)
$$

and for each $\omega \in \Omega$, and $t \geq T$, and $s \leq t$, one can prove $|Y(s)| \leq \frac{\alpha \varepsilon t}{4}$, see [13]. It follows that for $\omega \in \Omega_{T}$, and $t \geq T$,

$$
2 \alpha \hat{z}(t) e^{-\frac{1}{4} \alpha \varepsilon t} \leq z(t) \leq 2\left(x^{-2}+2 \alpha\right) \hat{z}(t) e^{-\frac{1}{4} \alpha \varepsilon t},
$$


together with (2.12), implies that

$$
\left(2 \alpha \beta-k_{m}^{2}-\frac{1}{2} \alpha \varepsilon\right) t+C_{1} \leq \log z(t) \leq\left(2 \alpha \beta-k_{m}^{2}+\frac{1}{2} \alpha \varepsilon\right) t+C_{2} .
$$

It follows from (2.4) that for $\omega \in \Omega$ and $t \geq T$,

$$
\beta-\frac{k_{m}^{2}}{2 \alpha}-\frac{\varepsilon}{2}+\frac{C_{1}-\log \left(x^{-2}\right)}{\alpha t} \leq \frac{1}{t} \int_{0}^{t} X^{2}(s) d s \leq \beta-\frac{k_{m}^{2}}{2 \alpha}+\frac{\varepsilon}{2}+\frac{C_{2}-\log \left(x^{-2}\right)}{\alpha t_{0}} .
$$

By the definition of $t_{0}$, we have for $\omega \in \Omega$ and $t \geq T$,

$$
\beta-\frac{k_{m}^{2}}{2 \alpha}-\varepsilon \leq \frac{1}{t} \int_{0}^{t} X^{2}(s) d s \leq \beta-\frac{k_{m}^{2}}{2 \alpha}+\varepsilon,
$$

which is the desired result when $\alpha \beta>\frac{k_{m}^{2}}{2}$. If $\alpha \beta=\frac{k_{m}^{2}}{2}$, we let $\hat{\beta}=\beta+\epsilon$ and then we get $\alpha \hat{\beta}>\frac{k_{m}^{2}}{2}$

$$
\hat{\beta}-\frac{k_{m}^{2}}{2 \alpha}-\varepsilon \leq \frac{1}{t} \int_{0}^{t} X^{2}(s) d s \leq \hat{\beta}-\frac{k_{m}^{2}}{2 \alpha}+\varepsilon .
$$

Letting $\epsilon \rightarrow 0$, we arrive that $X(t) \rightarrow 0$ almost surely as $t \rightarrow \infty$.

When $\alpha \beta<\frac{k_{m}^{2}}{2}$, it follows from the following property of Brownian motion ([8])

$$
\lim \sup _{t \rightarrow \infty} \frac{B_{t}}{\sqrt{2 t \log \log t}}=1 \quad \text { a.s. }
$$

that $X(t) \rightarrow 0$ almost surely as $t \rightarrow \infty$. The proof of Theorem 2.1 is complete.

Remark 2.1. From Theorem 2.1, it follows that the weak noise can change the value of equilibrium and the strong noise can destroy the stability of equilibrium.

\section{COMPETITION BETWEEN NONLINEAR TERM AND NOISE TERM}

In this section, we consider the role of competition between nonlinear term and noise term. Before that, we first list out what type of noise can make the solution of (1.1) keep positive.

$$
d X_{t}=f\left(X_{t}\right) d t+\sigma\left(X_{t}\right) d W_{t}, \quad X_{0}=x
$$

Using the test function (see [1])

$$
\psi_{k}(r)= \begin{cases}0, & (-\infty, 0], \\ \frac{2 k^{2} r^{3}}{3}, & {\left[0, \frac{1}{2 k}\right],} \\ r-\frac{1}{2 k}-\frac{2 k^{2}}{3}\left(r-\frac{1}{k}\right)^{3}, & {\left[\frac{1}{2 k}, \frac{1}{k}\right],} \\ r-\frac{1}{2 k}, & {\left[\frac{1}{k}, \infty\right),}\end{cases}
$$

and Itô formula, it is not hard to get the following Proposition. 
Proposition 3.1. Assume that the function $f(r)$ is continuous on $\mathbb{R}$ and such that $f(r) \geq 0$ for $r \leq 0$ and $\sigma(r)$ satisfies the local Lipschitz condition, i.e., there exists constant $m>1$ such that $|\sigma(x)| \leq l_{\sigma}|x|^{m}$, where $l_{\sigma}$ is the local Lipschitz constant. Then the solution of (3.1) with nonnegative initial datum remains positive, i.e., $X_{t} \geq 0$, a.s., $t \geq 0$.

Consider the following problem

$$
\left\{\begin{array}{l}
d X_{t}=\left(-k_{1} X_{t}^{\gamma}\right) d t+k_{2} X_{t}^{m} d W_{t}, \quad t>0, \\
X_{0}=x
\end{array}\right.
$$

where $k_{1} \geq 0, k_{2} \in \mathbb{R}, m \geq 1$ and $\gamma>1$ satisfying $(-1)^{\gamma}=-1$. When $k_{1} \geq 0$ and $(-1)^{\gamma}=-1$, the existence of local solution of $(3.2)$ can be obtained by Picard iteration, see $[5,9,10]$. When $(-1)^{\gamma}=1$ and $k_{1}<0$, the solution of $(3.2)$ will blow up in finite time, see $[3,4,7]$.

Theorem 3.1. Assume that $m>\frac{1+\gamma}{2}, x$ is a nonnegative constant satisfying

$$
\frac{k_{2}^{2}}{2} x^{2 m}>\frac{2 m-(1+\gamma)}{2 m}\left(\frac{1+\gamma}{m k_{2}^{2}}\right)^{\frac{1+\gamma}{2 m-(1+\gamma)}}\left(2 k_{1}\right)^{\frac{2 m}{2 m-(1+\gamma)}} .
$$

Then the solution of (3.2) will blow up in finite time in $L^{2}(\Omega)$, that is, there exists a constant $T^{*}>0$ such that

$$
\lim _{t \rightarrow T^{*}-0}\left(\mathbb{E}\left|X_{t}\right|^{2}\right)^{\frac{1}{2}}=\infty .
$$

Proof. It follows from Proposition 3.1 that the solution $X_{t} \geq 0$ holds almost surely. By Itô formula, we have

$$
X_{t}^{2}=x^{2}-2 k_{1} \int_{0}^{t} X_{s}^{\gamma+1} d s+2 k_{2} \int_{0}^{t} X_{s}^{m+1} d W_{s}+k_{2}^{2} \int_{0}^{t} X_{s}^{2 m} d s .
$$

Taking expectation on both sides of the above equality and letting $\xi(t)=\mathbb{E}\left[X_{t}^{2}\right]$, we have

$$
\xi(t)=x^{2}-2 k_{1} \mathbb{E} \int_{0}^{t} X_{s}^{\gamma+1} d s+k_{2}^{2} \mathbb{E} \int_{0}^{t} X_{s}^{2 m} d s,
$$

or, in the differential form

$$
\left\{\begin{array}{l}
\frac{d \xi(t)}{d t}=-2 k_{1} \mathbb{E}\left[X_{t}^{\gamma+1}\right]+k_{2}^{2} \mathbb{E}\left[X_{t}^{2 m}\right], \\
\xi(0)=x^{2}
\end{array}\right.
$$

By Jensen's inequality, we have

$$
\mathbb{E}\left[X_{s}^{\gamma+1}\right] \leq\left[\mathbb{E} X_{s}^{2 m}\right]^{\frac{1+\gamma}{2 m}}, \quad \mathbb{E}\left[X_{t}^{2 m}\right] \geq\left(\mathbb{E}\left[X_{t}^{2}\right]\right)^{m}
$$


and $\varepsilon$-Young's inequality yields

$$
2 k_{1}\left(\mathbb{E}\left[X_{s}^{2 m}\right]\right)^{\frac{1+\gamma}{2 m}} \leq \frac{k_{2}^{2}}{2} \mathbb{E} X_{s}^{2 m}+\tilde{k}_{1},
$$

where $\tilde{k}_{1}=\frac{2 m-(1+\gamma)}{2 m}\left(\frac{1+\gamma}{m k_{2}^{2}}\right)^{\frac{1+\gamma}{2 m-(1+\gamma)}}\left(2 k_{1}\right)^{\frac{2 m}{2 m-(1+\gamma)}}$. Submitting (3.6) and (3.7) into (3.5), we get

$$
\left\{\begin{array}{l}
\frac{d \xi(t)}{d t} \geq \frac{k_{2}^{2}}{2} \xi^{m}(t)-\tilde{k}_{1} \\
\xi(0)=x^{2}
\end{array}\right.
$$

This implies that, for $\frac{k_{2}^{2}}{2} \xi^{m}(0)-\tilde{k}_{1}>0$, we have $\frac{k_{2}^{2}}{2} \xi^{m}(t)-\tilde{k}_{1}>0$ and $\xi(t)>\xi_{0}$, for $t>0$. An integration of equation (3.8) gives that

$$
\begin{aligned}
T & \leq \int_{\xi(0)}^{\xi(T)} \frac{2 d r}{k_{2}^{2} r^{m}-2 \tilde{k}_{1}} \\
& \leq \int_{\xi(0)}^{\infty} \frac{2 d r}{k_{2}^{2} r^{m}-2 \tilde{k}_{1}} \\
& <\infty,
\end{aligned}
$$

which implies that $\eta(t)$ must blow up at a time $T^{*} \leq \int_{\xi(0)}^{\infty} \frac{2 d r}{k_{2}^{2} r^{m}-2 \tilde{k}_{1}}$. This completes the proof.

Next, we consider the case that $1<m<\frac{1+\gamma}{2}$.

Theorem 3.2. Assume that $1<m<\frac{1+\gamma}{2}$ and $x$ is a nonnegative constant. Then (3.2) has a global solution.

Proof. It follows from $[5,10,16]$ that $(3.2)$ has a local solution on $[0, T]$. By Proposition 3.1, this local solution is positive. Now, we prove the solution does not blow up in finite time. Similar to the proof of Theorem 3.1, we have

$$
\left\{\begin{array}{l}
\frac{d \xi(t)}{d t}=-2 k_{1} \mathbb{E}\left[X_{t}^{\gamma+1}\right]+k_{2}^{2} \mathbb{E}\left[X_{t}^{2 m}\right], \\
\xi(0)=x^{2}
\end{array}\right.
$$

where $\xi(t)=\mathbb{E}\left[X_{t}^{2}\right]$. By Hölder inequality and $\varepsilon$-Young's inequality, we have

$$
\begin{aligned}
\mathbb{E}\left[X_{t}^{2 m}\right] & \leq\left(\mathbb{E}\left[X_{t}^{1+\gamma}\right]\right)^{\frac{2(m-1)}{\gamma-1}}\left(\mathbb{E}\left[X_{t}^{2}\right]\right)^{\frac{1+\gamma-2 m}{\gamma-1}} \\
& \leq k_{1} \mathbb{E}\left[X_{t}^{1+\gamma}\right]+\hat{k}_{1} \mathbb{E}\left[X_{t}^{2}\right]
\end{aligned}
$$

where

$$
\hat{k}_{1}=\frac{1+\gamma-2 m}{\gamma-1}\left(\frac{k_{1}(\gamma-1)}{2(m-1)}\right)^{\frac{2(m-1)}{1+\gamma-2 m}}\left(k_{2}^{2}\right)^{\frac{\gamma-1}{1+\gamma-2 m}}
$$


Submitting (3.10) into (3.9), we get

$$
\begin{aligned}
\frac{d \xi(t)}{d t} & \leq-k_{1} \mathbb{E}\left[X_{t}^{\gamma+1}\right]+\hat{k}_{1} \xi(t) \\
& \leq \hat{k}_{1} \xi(t)
\end{aligned}
$$

which yields that

$$
\xi(t) \leq \xi(0) e^{\hat{k}_{1} t}
$$

Suppose $\zeta$ is the lifetime of $X(t)$. Define

$$
\tau_{R}=\inf \left\{t>0, \quad X^{2}(t) \geq R\right\}, \quad R>0,
$$

It is clear that $\tau_{R}$ tends to the lifetime $\zeta$ as $R \rightarrow+\infty$. (3.11) implies that

$$
\mathbb{E}\left[X^{2}\left(t \wedge \tau_{R}\right)\right] \leq \mathbb{E}\left[X^{2}(0)\right] e^{\hat{k}_{1} t} .
$$

Letting $R \rightarrow+\infty$ in above inequality, by Fatou lemma, we get

$$
\mathbb{E}\left[X^{2}(t \wedge \zeta)\right] \leq \mathbb{E}\left[X^{2}(0)\right] e^{\hat{k}_{1} t}
$$

Now if $P(\zeta<+\infty)>0$, then for a large $T>0, P(\zeta \leq T)>0$. Taking $t=T$ in (3.12), we get

$$
\mathbb{E}\left[\mathbf{1}_{\zeta \leq T} X^{2}(\zeta)\right] \leq \mathbb{E}\left[X^{2}(0)\right] e^{\hat{k}_{1} t}
$$

Since $X^{2}(\zeta)=\infty$ on a positive measure subset $\zeta \leq T$, the left hand side of (3.13) is infinite, while the right hand side is finite, which is impossible. Therefore $P(\zeta=$ $+\infty)=1$.

Remark 3.1. Combining Theorems 3.1 and 3.2, we find the competition between the nonlinear term and noise term. The value $m=(1+\gamma) / 2$ is a threshold for equation (3.2). For example, considering the equation (3.2) with $\gamma=3$, we have the following results. When $1 \leq m<2$, equation (3.2) has a global solution; when $m>2$, the solution of equation (3.2) will blow up in finite time; when $m=2$, it follows from the proofs of Theorems 3.1 and 3.2 that the solution of equation (3.2) will blow up in finite time if $k_{2}^{2}>2 k_{1}$ and equation (3.2) has a global solution if $k_{2}^{2} \leq 2 k_{1}$.

\section{SIMULATIONS}

In this section, we give some simulations to illustrate the results of Theorems 2.1, 3.1 and 3.2. Firstly, taking the initial date $x=0.1, \alpha=1, \beta=2$ and $k(t)=\sqrt{2}$, we have 


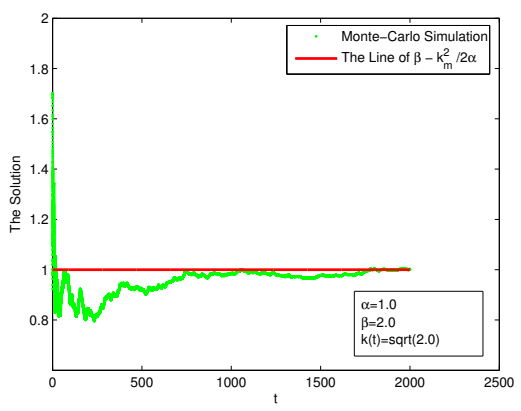

Figure 1: The case that $\alpha \beta>k_{m}^{2} / 2$

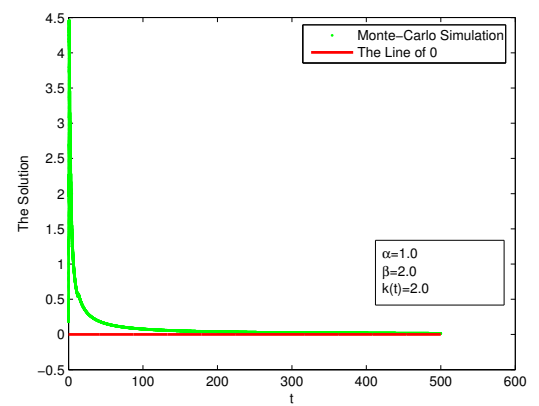

Figure 3: The case that $\alpha \beta=k_{m}^{2} / 2$

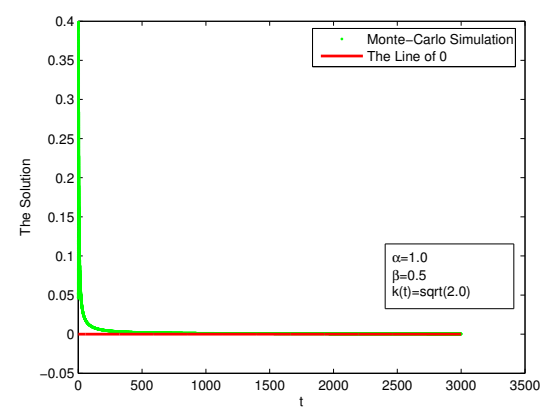

Figure 2: The case that $\alpha \beta<k_{m}^{2} / 2$

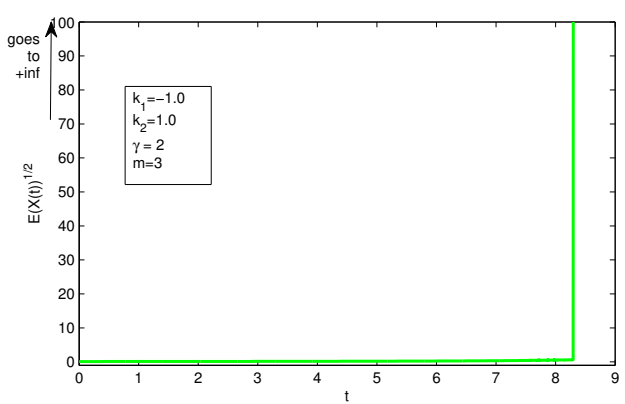

Figure 4: The case that $k_{1}<0$ and $(-1)^{\gamma}=1$

$\alpha \beta>\frac{k_{m}^{2}}{2}$. It follows Theorem 2.1 that $\frac{1}{t} \int_{0}^{t} X_{s}^{2} d s \rightarrow \beta-\frac{k_{m}^{2}}{2 \alpha}$, see Fig 1 . Under the same initial data, taking $\alpha=1, \beta=0.5, k(t)=\sqrt{2}$, and $\alpha=1, \beta=2$ and $k(t)=2$, we have $\alpha \beta<\frac{k_{m}^{2}}{2}$ and $\alpha \beta=\frac{k_{m}^{2}}{2}$, respectively. It follows from Theorem 2.1 that the solution goes to 0 as times goes to infinity, see Figs 2 and 3.

In order to verify the results of Theorems 3.1 and 3.2 , we take the initial data $x=2$ holds for Figs 4 -8. It is easy to verify that the condition (3.3) holds for $x=2$, $k_{1}=k_{2}=1$ or $k_{1}=1, k_{2}=2$. First we note that if $k_{1}<0$ and $\gamma>1$, then the solution of (3.2) will blow up in finite time in $L^{2}(\Omega)$, see Fig 4. Theorem 3.1 shows that if $m>\frac{1+\gamma}{2}$, the solution of $(3.2)$ will blow up in finite time in $L^{2}(\Omega)$, see Fig 5. Theorem 3.2 shows that if $m<\frac{1+\gamma}{2}$, the solution of (3.2) exist globally, see Fig 6. When $m=\frac{1+\gamma}{2}$, from Remark 3.1, the solution of equation (3.2) will blow up in finite time if $k_{2}^{2}>2 k_{1}$ (see Fig 7) and equation (3.2) has a global solution if $k_{2}^{2} \leq 2 k_{1}$, see Fig 8. 


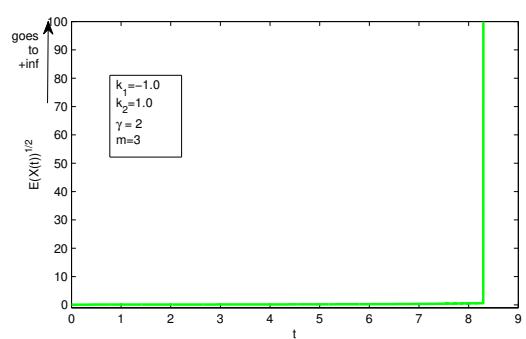

Figure 5: The case that $m>\frac{1+\gamma}{2}$

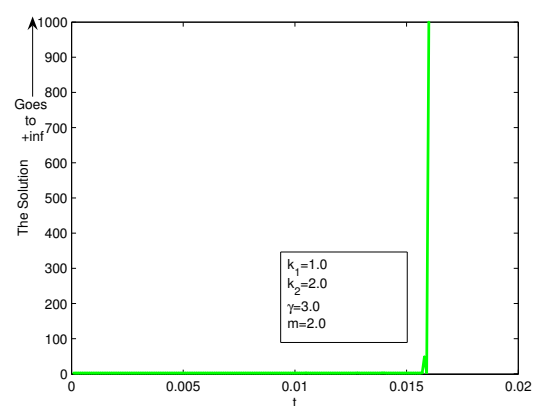

Figure 7: The case that $m=\frac{1+\gamma}{2}$ and $k_{2}^{2}>2 k_{1}$

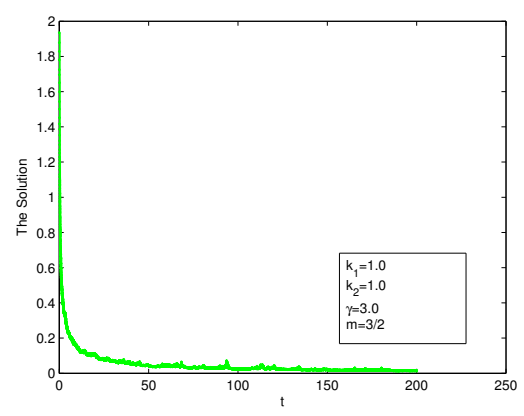

Figure 6: The case that $m<\frac{1+\gamma}{2}$

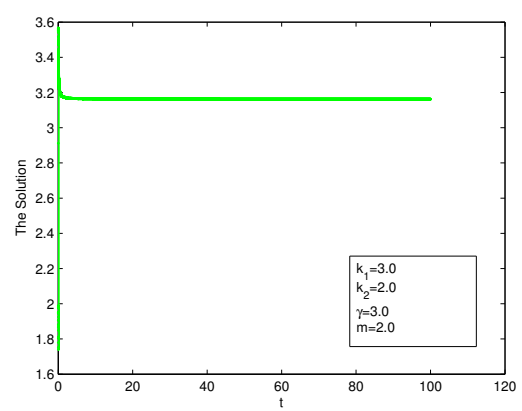

Figure 8: The case that $m=\frac{1+\gamma}{2}$ and $k_{2}^{2}<2 k_{1}$

\section{ACKNOWLEDGMENTS}

The first author was supported in part by NSFC of China grants 11771123 and 11301146, the Project Funded by China Postdoctoral Science Foundation (Grant No. 2016M600427) and Postdoctoral Science Foundation of Jiangsu Province (Grant No. 1601141B).

\section{REFERENCES}

[1] J. Bao and C. Yuan, Blow-up for stochastic reactin-diffusion equations with jumps, J. Theor. Probab., 29 (2016), 617-631.

[2] Z. Cheng, J.Q. Duan, and L. Wang, Most probable dynamics of some nonlinear systems under noisy fluctuations, Commun. Nonlinear Sci. Numer. Simul., 30, (2016) 108-114. 
[3] P-L. Chow, Unbounded positive solutions of nonlinear parabolic Itô equations, Communications on Stochastic Analysis, 3 (2009), 211-222.

[4] P-L. Chow and R. Khasminskii, Almost sure explosion of solutions to stochastic differential equations, Stochastic Process. Appl., 124 (2014), 639-645.

[5] S. Z. Fang and T. S. Zhang, A study of a class of stochastic differential equations with non-Lipschizian coefficients, Probab. Theory Related Fields, 132, (2005) 356-390.

[6] M. Hofmanova and J. Seidler, On weak solutions of stochastic differential equations, Stoch. Anal. Appl., 30 (2012), 100-121.

[7] I. Ikeda and S. Watanabe, Stochastic Differential Equations and Diffusion Processes, North-Holland, Amsterdam, 1981.

[8] J. Lamperti, Stochastic Processes, Springer-Verlag, 1977, ISBN 978-1-4684-93580 .

[9] G. Q. Lan, Pathwise uniqueness and non-explosion of stochastic differential equations with non-Lipschitzian coefficients, Acta Math. Sinica (Chin. Ser.), 52 (2009), 109-114.

[10] G. Q. Lan and J. L. Wu, New sufficient conditions of existence, moment estimations and non confluence for SDEs with non-Lipschitzian coefficients, Stochastic Process. Appl., 124 (2014), 4030-4049.

[11] H.P. McKean, Stochastic Integrals, New York, Academic Press, ISBN: 978-14832-59239.

[12] M. Niu and B. Xie, Impacts of Gaussian noises on the blow-up times of nonlinear stochastic partial differential equation, Nonlinear Anal. Real World Appl., 13 (2012), 1346-1352.

[13] B. Øksendal, G. Våge, and H. Zhao, Two properties of stochastic KPP equation: ergodicity and pathwise property, Nonlniarity, 14 (2001), 639-662.

[14] J. Shao, F.-Y. Wang, and C. Yuan, Harnack inequalities for stochastic (functional) differential equations with non- Lipschitzian coefficients, Electron. J. Probab., 17, No. 18 (2012).

[15] D. W. Stroock and S. R. S. Varadhan, Multidimensional Diffusion Processes, Springer-Verlag, 1979.

[16] B. Xie, Some effects of the noise intensity upon non-linear stochastic heat equations on [0,1], Stochastic Process. Appl., 126 (2016), 1184-1205. 